\title{
Occupational Stress among Radiographers Working in Tertiary Care Hospital in Udupi and Mangalore
}

\author{
Kaushik Nayak ${ }^{1}$, Navish Kumar ${ }^{1}$, Nitika C. Panakkal ${ }^{3}$ \\ ${ }^{1}$ MSc Medical Imaging Technology, Department of Medical Imaging Technology, ${ }^{3}$ MSc Medical Imaging \\ Technology, Assistant Professor (Sr. Scale), Department of Medical Imaging Technology, Manipal College of \\ Health Professions, Manipal Academy of Higher Education, Manipal, Karnataka, India
}

\begin{abstract}
Introduction: Occupational stress can negatively impact an individual and is associated with a wide range of physical and mental effects resulting in psychosocial and biomechanical stress. Moreover, health care workers have been reported to have a great deal of stress mainly due to the shift duties and increased workload.
\end{abstract}

Objectives: Therefore the study aimed to determine the psychosocial predictors of stress among radiographers in tertiary care hospitals of Udupi and Mangalore. Data was collected from 55 radiographers from three tertiary care hospitals using a standard questionnaire published by the UK health and safety (HSE). A score and an aggregate score was generated for each item as well as each of seven sets of working condition like "job demands, control, support from managers, support from peers, role, change and relationships". The analysis tool published by the HSE was used to analyze data.

Results: The indicator analysis tool recommended 11 "psychosocial work conditions" that required improvement. On an aggregate level from the seven psychosocial working conditions 'relationship' and 'Demand' category warranted improvement.

Conclusion: The study showed majority categories being identified as "good and need to maintain performance". However that main predictors of occupational stress was found to be excess workload followed by relationship conflicts among co-workers.

Keywords: Occupational stress, Radiographers, Psychosocial stress, stressors, management standards.

\section{Introduction}

Occupational stress has been recognized as an important health problem for employees worldwide. The "health and safety executive "defines occupational

\section{Corresponding Author:}

Nitika C. Panakkal

Assistant professor (Sr. Scale), Department of Medical

Imaging Technology, Manipal College of Health

Professions, Manipal Academy of Higher Education

Manipal

Telephone: $91+8861615165$

e-mail: nitikacp@gmail.com; nitika.cp@manipal.edu stress as the "adverse reaction people have to excessive pressures or other demands placed upon them"(1). The combination of high demands over the job and a low amount of control over the situation can lead to stress. Occupational stress is considered as an issue of concern as it is been associated to a variety of adverse physical and mental effects resulting in biomechanical as well psychosocial stress ${ }^{(2,3)}$. Physical effects include Physical fatigue, respiratory problems, headaches, low back pain and muscular tension and impaired immune functioning ${ }^{(4)}$. Mental effects include mood swings, anxiety and also socially withdrawn which results in lowered performance and productivity, obesity or weight loss and increased intake of alcohol ${ }^{(4)}$.Sometimes a more severe consequence is "burnout". Burnout is 
considered a form of emotional and mental exhaustion, depersonalization and reduced sense of personal accomplishment ${ }^{(5)}$. Stress in workplace can have many different sources that can effect both the employer and employees involved. The factors that causes stress is called stressors. According to Health and safety executive management standards there are 6 work design stressors that if not managed well can result in a decreased productivity, poor health and wellbeing. These stressors are Demand, control, support, role, relationships and change ${ }^{(1)}$. Among the various health care professionals, radiographers in general are exposed to a great deal of stress where work problems, role ambiguity, role conflict and social support problems is considered the highest predictors of stress ${ }^{(6)}$. Occupational stress if present in the workplace should be managed for improving individual mental and physical health thus improving work efficiency and productivity for better patient care. Although numerous studies have explored work stress among health care professionals in many countries, there are few in the country that is been done on radiographers. To the best of our knowledge there is no published data to evaluate the occupational stress among radiographers in the Udupi and Mangalore region. Therefore the aim of the study was to determine the psychosocial predictors of stress among radiographers in tertiary care hospitals of Udupi and Mangalore.

\section{Material and Method}

The study approval was acquired from Institution Research Committee and ethics committee. The sample size included 55 radiographers from 3 tertiary care hospitals of Udupi and Mangalore regions. Data was collected using a self-administered questionnaire published by health and safety executive $\mathrm{UK}^{(7)}$. A pretest was conducted among 3 radiographers to know whether they were able to understand the questions. The questionnaire included thirty five questions with a 5 point Likert scale (never, seldom, sometimes, often and always). Each item was represented by an individual score with a value ranging from 1-5 with 1 being the least desirable and 5 is the most desirable. The scoring was reversed for negatively phrased question. The questionnaire included two sections. The first focused on the individual s personal profile like subjects name, gender, years of experience and section two measured the psychosocial work conditions. The minimum and maximum score obtained on the questionnaire was considered 35 and 175 respectively. The data obtained from the questionnaire were analyzed using SPSS version 22.0. To analyze the data for possible stressors, the questionnaire responses were entered into an excel sheet analysis tool published by HSE management standards. Individual score and an aggregate score was generated for each item as well as for each of 7 sets of psychosocial working condition like "job demands, control, Manager Support, peer support, role, change and relationships". The analysis tool also presents recommendation for action in relation to benchmark data by assigning color code. The four categories of recommendation include "red: urgent action needed", "yellow: clear need for improvement", "blue: good, but need for improvement" and "green: doing very well, need to maintain performance". Descriptive analysis was done to summarize the average levels of job stress. Independent $t$ test was used to find out any differences in stress levels between various stressors among Gender, age groups $(<40$ years and $>40$ years), work experience $(<2$ years and $>2$ years) and marital status (married and unmarried). A $p$ value $<0.05$ was considered significant when comparing the means between various groups.

\section{Results}

The study included 55 radiographers from 3 different tertiary care hospitals. In our study $20 \%$ of radiographers were above 40 years of age. Majority of them were males (55\%), with females comprising of $45 \%$. Out of the 55 radiographers, $51 \%$ of them were married. 22 out of 55 radiographers had less than 10 years of experience and $25 \%$ of them had less than 2 years of experience. The mean score for job stress among 55 radiographer was found to be 133 ranging from 105 to 163 [table 1]. The mean score of indicator items for the seven stressors is depicted in figure 1 . The mean score identified below the $20^{\text {th }}$ percentile in comparison to the benchmark data gathered by HSE from 136 organizations was "red lighted" "by the analysis tool indicating "urgent action required". None of the 7 management standards categories was found to be below the $20^{\text {th }}$ percentile at an aggregate level. However, scores of 3 individual items dispersed across 2 stressor category were below the $20^{\text {th }}$ percentile indicating urgent need of action. Scores on 3 items were yellow lighted as they were found to be between the $24^{\text {th }}$ and $49^{\text {th }}$ percentile indicating "clear need for improvement". These items were scattered across 2 of the analysis categories. At the aggregate level, one among the seven categories (relationships) were "yellow lighted". Scores on 5 items were found to be between the $50^{\text {th }}$ and the $79^{\text {th }}$ percentile. These items were highlighted blue with the recommendation 
"good, but need for improvement" and were distributed across the 'Demand and Control 'category. At the aggregate level, one among seven categories (demands) was observed as being in this group. Majority of the items (24) were identified as being greater than the $80^{\text {th }}$ percentile and were green lighted indicating 'doing very well, need to maintain performance'. In sum, out of 35 psychosocial working conditions examined through the indicator tool, 11 of them indicated requiring improvement when compared to benchmark data gathered from organizations in Britain.
Independent $t$ test showed no significant difference in mean scores for all seven analysis categories between males and females. " $(\mathrm{p}=0.38 ; \mathrm{p}=0.49 ; \mathrm{p}=0.60 ; \mathrm{p}$ $=0.87 ; \mathrm{p}=0.12 ; 0.77 ; 0.87)$ ". Similarly there was no significant difference in mean score of stress levels among age groups " $(\mathrm{p}=0.47 ; \mathrm{p}=0.91 ; \mathrm{p}=0.50 ; \mathrm{p}=$ $0.75 ; \mathrm{p}=0.64 ; 0.50 ; 0.53)$ ", years of experience " $(\mathrm{p}=$ $0.14 ; p=0.99 ; p=0.38 ; p=0.82 ; p=0.35 ; p=0.75 ; p=$ $0.23)$ " and marital status " $(p=0.36 ; p=0.05 ; p=0.24$; $\mathrm{p}=0.911 ; \mathrm{p}=0.64 ; \mathrm{p}=0.98 ; \mathrm{p}=0.78)$ ” for all seven analysis categories.

Table 1: Mean, Standard deviation, minimum and maximum levels of job stress among Radiographers.

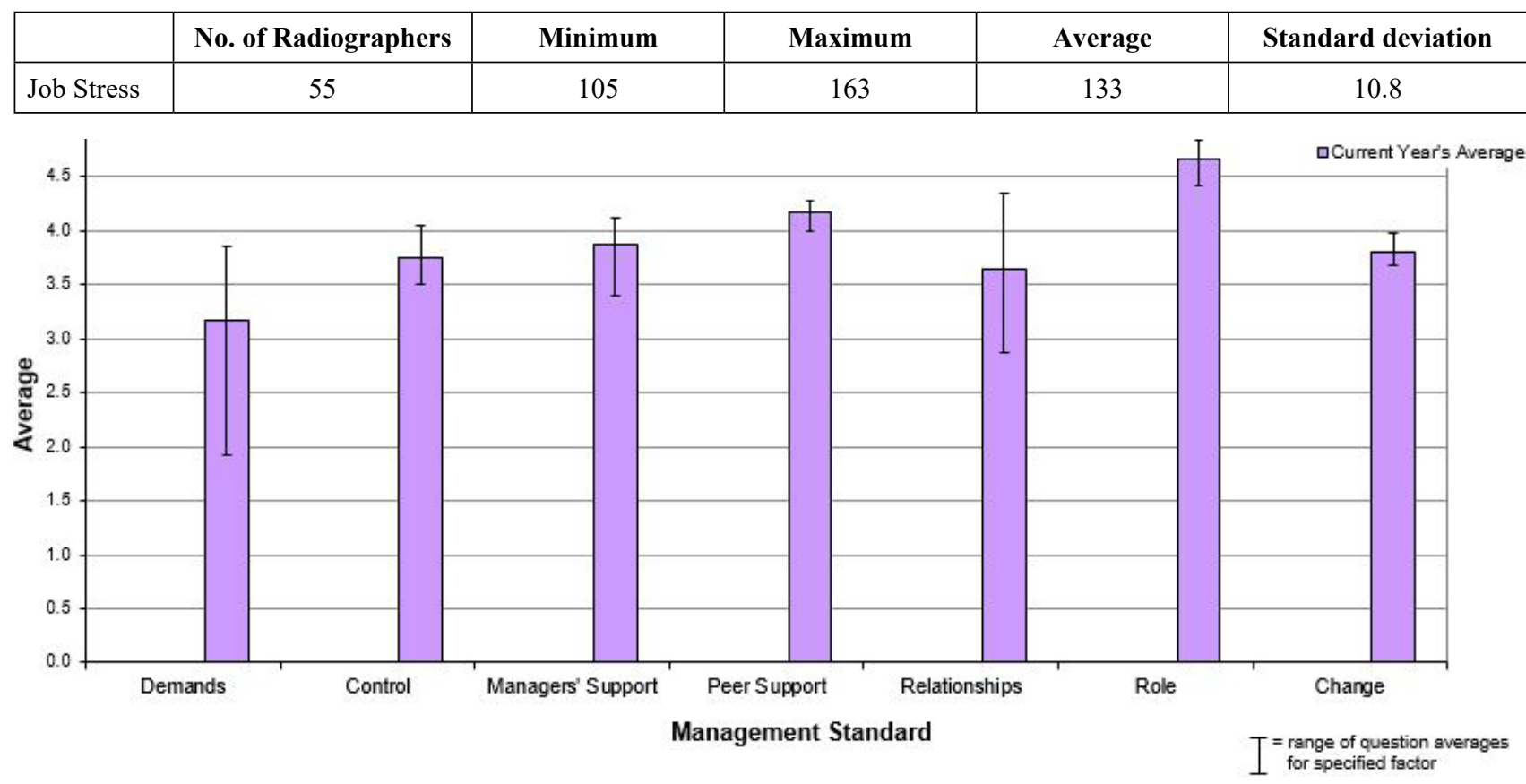

Figure 1. Bar diagram showing the mean score of indicator items for seven stressor categories

\section{Discussion}

The results of the study showed that 24 out of 35 items were recognized as being greater than $80^{\text {th }}$ percentile indicating 'doing very well and need to maintain performance and the rest of 11 evaluated through indicator tool were indicated as requiring improvement when compared with "HSE s current benchmark data". A lot of studies report excess demand, lack of social support, role conflict and managerial support as the main sources of occupational stress among radiographers ${ }^{(3,6,8,9)}$. Although the present study shows that majority of the categories of psychosocial work environment is good and need to be maintained, there is a need for improvement for the demand and relationship category. Demand can be explained as those aspects of the job in a working environment that is difficult for the employee to achieve ${ }^{(4)}$. These include workload, work pattern and working environment. A study conducted by Natal el al reported that accelerated work load and faulty equipment was the major cause of occupational stress. The high workload was due to high patient to staff ratio requiring more effort from radiographers to work more hard and intensively to meet the demands of staff shortage and high workload ${ }^{(4)}$. The present study showed that majority $(70 \%)$ of the respondents agreed that they had to work very intensively and very fast more often to 
compensate for staff shortage and high workload. The relationship stressor is due to dealing with unacceptable behavior and is caused when a person is subjected to "personal harassment in the form of unkind words or behavior". Conflict in the workplace has been recognized as a significant cause of stress for some workers ${ }^{(10)}$. In a survey conducted by Guest et al in 2004, $13 \%$ of participants had experienced bullying or harassment by co-workers ${ }^{(11)}$. The present study reported that around $44 \%$ of staff responded that they are always, often and sometimes bullied and $24 \%$ staff reported that they are always, often or sometimes subjected to "personal harassment in form of unkind words or behavior". These recommendations provided by the indicator analysis tool are made basedon benchmark data obtained from a representative number of professional groups and are not organization or sector specific. Therefore further studies can be done to develop a standardized questionnaire to evaluate the occupational stress among health care professionals. Interventions can be done for staff members to attend workshops that would help develop their skills and attitude and the effectiveness of these interventions should be assessed in response to the analysis tool recommendation. One of the limitations of the study was that there was a low response rate, as a result only few radiographers enrolled for the study. Therefore future studies can be done in a large scale to include more number of hospitals locally or regionally. Also the biomechanical stress was not evaluated in the present due to time constraints. Musculoskeletal stress is often related to excess workload which can result in psychosocial stress $^{(3)}$. Therefore further research can be done to evaluate the biomechanical stress among radiographers. Managing occupational stress will not only improve the work efficiency but also increase the productivity which in turn will result in a better quality of care in healthcare.

\section{Conclusion}

The study has shown the main source of stress being excess demand followed by relationship conflicts among co- workers. Therefore it is recommended to develop interventions needed to manage psychosocial stress that may result in an improved work efficiency.

Acknowledgement: The authors would like to acknowledge the Institute research committee and ethics committee, Kasturba hospital for their support to undertake this study. We also extend our heartfelt gratitude to all the participating organizations for their support in data collection. We are also extremely thankful to Health and safety executive UK for providing us with the data collection tool.

\section{Conflict of Interest: None}

Funding: There no external funding for this research project as this project was conducted within the institute itself that provided us with all facilities required to conduct the project.

\section{Ethical Clearance: Yes}

\section{References}

1. Sunley K. work related stress guidance for health and safety representatives [Internet]. First edit. London: society of radiogrpahers; 2007. 1-44 p. Available from: file://E:/intern projects/navish and kaushik/occupational stress among radiographers/ sor_work_related_stress_guidance_health.pdf

2. R CG, Bekezela N. The prevalence and antecedents of occupational stress among radiographers in Zimbabwe: Interplay of economics and culture. Int J Med Res Heal Sci. 2013;2(2):233.

3. Ugwu AC, Egwu OA, Ochie K, Ewunonu EO, Ovuoba KN, Njoku CO. Incidence of occupational stress among medical radiographers: a population based zonal survey. Niger J Physiol Sci. 2007;22(12):123-7.

4. Gam NP. Occupational stressors in diagnostic Radiographers working in public health facilities in Ethekwini District of Kwazulu-Natal [Internet]. Vol. 5. Durban University of Technology; 2012. Available from: https://ir.dut.ac.za/ handle/10321/1414

5. Braithwaite M. Nurse Burnout and Stress in the NICU. Adv Neonatal Care [Internet]. 2008 Dec [cited 2019 Jun 26];8(6):343-7. Available from: http://www.ncbi.nlm.nih.gov/pubmed/19060580

6. Ugwu AC, Erondu OF, U B Umeano. Psychosocial stress and its predictors among radiographers in south-eastern Nigeria. South African Radiogr. 2011;49(2):11-5.

7. Bevan A, Houdmont J, Menear N. The management standards indicator tool and the estimation of risk. Occup Med (Chic Ill). 2010;60(7):525-31.

8. Pas H. Assessment of occupational stress level among the diagnostic radiographers of Kandy District in Sri Lanka. Int J Perceptions Public Heal. 2017;1(3):205-9. 
9. Rutter DR, Lovegrove MJ. Occupational stress and its predictors in radiographers. Radiography. 2008;14(2):138-43.

10. Kendall E, $\mathrm{Ph} \mathrm{D}$, Neill VO. WORKERS , COMPENSATION AND REHABILITATION Occupational Stress : Factors that Contribute to its
Occurrence and Effective Management Centre for Human Services. 2000;

11. Guest DE, Conway N. Employee well-being and the psychological contract : a report for the CIPD. Chartered Institute of Personnel and Development; 2004. 